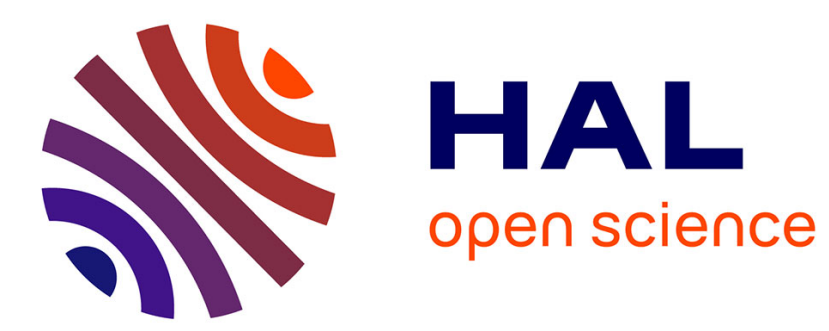

\title{
A Note on the Distribution of Some Additive Functions
}

Gérald Tenenbaum

\section{To cite this version:}

Gérald Tenenbaum. A Note on the Distribution of Some Additive Functions. Proceedings of the Steklov Institute of Mathematics, 2012, 276, pp.257-260. hal-01281340

\section{HAL Id: hal-01281340 https://hal.science/hal-01281340}

Submitted on 2 Mar 2016

HAL is a multi-disciplinary open access archive for the deposit and dissemination of scientific research documents, whether they are published or not. The documents may come from teaching and research institutions in France or abroad, or from public or private research centers.
L'archive ouverte pluridisciplinaire HAL, est destinée au dépôt et à la diffusion de documents scientifiques de niveau recherche, publiés ou non, émanant des établissements d'enseignement et de recherche français ou étrangers, des laboratoires publics ou privés. 


\title{
A note on the distribution of some additive functions
}

\author{
Gérald Tenenbaum \\ In memory of Anatolii Alekseevich Karatsuba, \\ with respect and admiration
}

\begin{abstract}
Let $f$ denote an additive arithmetical function with continuous limiting distribution $F$ on the integers. Then $f$ also has a limiting distribution $G$ on shifted primes. Under some growth conditions on the values of $f$ at primes, we provide optimal lower bounds for the modulus of continuity of $F$ and $G$, at all points from a specified infinite set.

Keywords: probabilistic number theory, Erdős-Wintner theorem, arithmetic functions, additive functions, concentration functions, distribution of arithmetic functions, modulus of continuity.
\end{abstract}

\section{Introduction}

The famous Erdős-Wintner theorem [6] states that a real, additive arithmetical function $f$ has a limiting distribution if, and only if, the following three series converge

$$
\sum_{|f(p)| \leqslant 1} \frac{\varphi(p)}{p}, \quad \sum_{|f(p)| \leqslant 1} \frac{f(p)^{2}}{p}, \quad \sum_{|f(p)|>1} \frac{1}{p},
$$

where, here and in the sequel, the letter $p$ generically denotes a prime number. Moreover, the characteristic function of the limit law $F(z)$ is the given by the formula

$$
\widehat{F}(\tau):=\int_{\mathbb{R}} \mathrm{e}^{i \tau z} \mathrm{~d} F(z)=\prod_{p}\left(1-\frac{1}{p}\right) \sum_{\nu \geqslant 0} \frac{\mathrm{e}^{i \tau f\left(p^{\nu}\right)}}{p^{\nu}} .
$$

It has been long conjectured that the same conditions are also necessary and sufficient for the existence of a limiting distribution on a sequence of shifted primes, i.e. for the weak convergence, as $x \rightarrow \infty$, of the distribution functions

$$
\frac{1}{\pi(x)} \sum_{\substack{p \leqslant x \\ f(p+a) \leqslant z}} 1
$$

to a distribution function $G(z)$ whenever $a$ is a non-zero integer. The sufficiency part of this conjecture was established by Kátai [8] using the Bombieri-Vinogradov theorem, whereas the more difficult necessary part, known as the Erdős-Kubilius conjecture, has been proved by Hildebrand [7]. In this setting, the characteristic function of the limit law takes the form

$$
\widehat{G}(\tau):=\prod_{p \nmid a}\left(1-\frac{1}{p-1}+\sum_{\nu \geqslant 1} \frac{\mathrm{e}^{i \tau f\left(p^{\nu}\right)}}{p^{\nu}}\right)
$$


In both cases, it follows respectively from classical results of Jessen-Wintner and of Lévy that the limiting distribution is always of pure type and that it is continuous if, and only if,

$$
\sum_{f(p) \neq 0} \frac{1}{p}=\infty
$$

In the classical situation (2), some attention has been focused along the years on the local behaviour of the limit law. We refer the reader to the recent work [2] for a review of results on the concentration function of the law $F$, and to the papers of Toulmonde [12], [13], [14], and Tenenbaum-Toulmonde [10] for progress in the study of the modulus of continuity in the case $f(n):=\log \{n / \varphi(n)\}$, where $\varphi$ denotes the Euler totient.

In the analogous problem for shifted primes, corresponding to formula (3), Deshouillers and Hassani [3] recently considered the question of differentiability of the distribution function $G(z)$ when $f(n)=\log \{n / \varphi(n)\}$, and showed that the left derivative is infinite at all points in $f\left(2 \mathbb{N}^{*}\right)$.

The main purpose of this note is to supply fairly general hypotheses implying that $F$ and $G$ both have infinite derivatives at all points from a specified infinite set, and indeed to provide explicit lower estimates for the moduli of continuity at these points.

We write $\mathbb{N}_{a}:=\left\{n \in \mathbb{N}^{*}:(n, a)=1\right\}$ and put $v:=1$ if $2 \nmid a, v:=0$ if $2 \mid a$.

Theorem 1. Let $\delta>0$ and $c:=\delta /(1+\delta)$. Under hypotheses (4) and

$$
f(p) \ll 1 /(\log p)^{1+\delta},
$$

we have, as $\varepsilon$ approaches 0 ,

$F(z+\varepsilon)-F(z-\varepsilon) \gg_{z} \varepsilon^{1-c} \quad\left(z \in f\left(\mathbb{N}^{*}\right)\right), \quad G(z+\varepsilon)-G(z-\varepsilon) \gg_{z} \varepsilon^{1-c} \quad\left(z \in f\left(2^{v} \mathbb{N}_{a}\right)\right)$

It is interesting to note that, in the example of a strongly additive function $f$ satisfying $f(p):=1 /(\log p)^{1+\delta}$ with $0<\delta<1$, we know from a result of Babu (see also Exercise 259 of [9], with solution in [11]) that $F$ is absolutely continuous. This cannot happen when $f(p) \ll 1 / p^{\delta}$, since a result of Erdős [4] then guarantees that $F$ is purely singular. Such property also holds for $G$, as can be shown by adapting the solution, given in [11], of Exercise 256 of [9].

We also remark that our result above provides, as a weak consequence, the estimates

$$
Q_{F}(\varepsilon) \gg \varepsilon^{1-c}, \quad Q_{G}(\varepsilon) \gg \varepsilon^{1-c} \quad(0<\varepsilon<1)
$$

for the concentration functions associated to $F$ and $G$ respectively. While the second appears to be new, the first of these lower bounds recovers that of Erdős and Kátai [5], stated in the case of strongly additive functions such that $f(p)=1 /(\log p)^{1+\delta}$ and which is optimal up to a factor $(\log \log 1 / \varepsilon)^{2}$ — see [2] for an improvement.

The author takes pleasure in expressing here warm thanks to Jean-Marc Deshouillers, for drawing his attention to this topic and fruitful subsequent exchanges, and to Régis de la Bretèche, for helpful discussions on this matter. 


\section{Proof of Theorem 1}

We restrict to the case of $G$ with $a= \pm 1$, since the cases of arbitrary $a$ and of $F$ are analogous. We may plainly assume $\delta<1$.

Given $y>2$, we let

$$
\widehat{G_{y}}(\tau):=\prod_{2<p \leqslant y}\left(1-\frac{1}{p-1}\right) \sum_{\nu \geqslant 1} \frac{\mathrm{e}^{i \tau f\left(2^{\nu}\right)}}{2^{\nu}} \prod_{2<p \leqslant y}\left(1+\frac{p-1}{p-2} \sum_{\nu \geqslant 1} \frac{\mathrm{e}^{i \tau f\left(p^{\nu}\right)}}{p^{\nu}}\right)
$$

denote the characteristic function of the distribution function

$$
G_{y}(z):=\prod_{2<p \leqslant y}\left(1-\frac{1}{p-1}\right) \sum_{\substack{P^{+}(n) \leqslant y \\ f(2 n) \leqslant z}} \frac{1}{k(2 n)}
$$

where $k$ is the multiplicative function defined by $k\left(2^{\nu}\right):=2^{\nu}(\nu \geqslant 1), k\left(p^{\nu}\right):=$ $(p-2) p^{\nu} /(p-1)(\nu \geqslant 1, p>2)$. We have

$$
\widehat{G_{y}}(\tau)=\widehat{G}(\tau)\left\{1+O\left(\tau /(\log y)^{1+\delta}\right)\right\} \quad\left(|\tau| \leqslant(\log y)^{1+\delta}\right) .
$$

Thus the Berry-Esseen inequality implies, for all $T \geqslant 1$,

$$
\sup _{z \in \mathbb{R}}\left|G(z)-G_{y}(z)\right| \ll Q_{G}\left(\frac{1}{T}\right)+\frac{T}{(\log y)^{1+\delta}},
$$

where $Q_{G}$ designates the concentration function associated to $G$. Selecting $T=T_{y}:=$ $(\log y)^{(1+\delta) / 2}$, we obtain

$$
\sup _{z \in \mathbb{R}}\left|G(z)-G_{y}(z)\right| \ll Q_{G}\left(\frac{1}{T_{y}}\right) .
$$

Now, for all $\varepsilon>0,2<v \leqslant y$, and letting $r$ denote an integer all of whose prime factors belong to $] v, y]$, we have

$$
\sum_{|f(r)|>\varepsilon} \frac{1}{k(r)} \leqslant \sum_{r} \frac{1}{k(r)} \mathrm{e}^{-(\log v)^{1+\delta}\{\varepsilon-|f(r)|\}} \ll \mathrm{e}^{-\varepsilon(\log v)^{1+\delta}} \frac{\log y}{\log v} .
$$

For fixed $z:=f(2 m)$, sufficiently small $\eta>0, \varepsilon>0$ and large $y$, we select $v:=$ $\exp \left\{(\eta \varepsilon)^{c-1}\right\}$ and thus obtain, with a suitable constant $K$,

$$
\begin{aligned}
\sum_{\substack{P^{+}(n) \leqslant y \\
|f(2 n)-z| \leqslant \varepsilon}} \frac{1}{k(2 n)} & \geqslant \frac{1}{k(2 m)} \sum_{|f(r)| \leqslant \varepsilon} \frac{1}{k(r)} \geqslant \frac{1}{k(m)}\left\{\prod_{v<p \leqslant y}\left(1+\frac{1}{p-2}\right)-K \mathrm{e}^{-1 / \eta} \frac{\log y}{\log v}\right\} \\
& \gg_{\eta} \frac{\varepsilon^{1-c} \log y}{k(m)} .
\end{aligned}
$$

Inserting back in (6) taking (7) into account, we get, with suitable $c_{0}>0, c_{1}>0$,

$$
G(z+\varepsilon)-G(z-\varepsilon) \geqslant \frac{c_{0} \varepsilon^{1-c}}{k(m)}-c_{1} Q_{G}\left(\frac{1}{T_{y}}\right)
$$


whence, letting $y$ tend to infinity,

$$
G(z+\varepsilon)-G(z-\varepsilon) \gg \frac{\varepsilon^{1-c}}{k(m)} .
$$

\section{References}

[1] G. J. Babu, Absolutely continuous distribution functions of additive functions $f(p)=(\log p)^{-a}$, $a>0$, Acta Arith. 26 no 4 (1974/75), 401-403.

[2] R. de la Bretèche \& G. Tenenbaum, Sur la concentration de certaines fonctions additives, Math. Proc. Camb. Phil. Soc., to appear.

[3] J.-M. Deshouillers \& M. Hassani, A note on the distribution function of $\varphi(p-1) /(p-1)$, J. Aust. Math. Soc., to appear.

[4] P. Erdős, On the smoothness of the asymptotic distribution of additive arithmetical functions, Amer. J. Math. 61 (1939), 722-725.

[5] P. Erdos \& I. Kátai, On the concentration of distribution of additive functions, Acta Sci. Math. (Szeged) $41 \mathrm{n}^{\text {os }} 3-4$ (1979), 295-305.

[6] P. Erdős \& A. Wintner, Additive arithmetical functions and statistical independence, Amer. J. Math. 61 (1939), 713-721.

[7] A. Hildebrand, Additive and multiplicative functions on shifted primes, Proc. London Math. Soc. (3) $59 \mathrm{n}^{\circ} 2$ (1989), 209-232.

[8] I. Kátai, On distribution of arithmetical functions on the set prime plus one, Compositio Math. 19 (1968), 278-289.

[9] G. Tenenbaum, Introduction à la théorie analytique et probabiliste des nombres, troisième édition, coll. Échelles, Belin, 2008, 592 pp.

[10] G. Tenenbaum \& V. Toulmonde, Sur le comportement local de la répartition de l'indicatrice d'Euler. Funct. Approx. Comment. Math. 35 (2006), 321-338.

[11] G. Tenenbaum, in collaboration with J. Wu, Exercices corrigés de théorie analytique et probabiliste des nombres, Cours spécialisés, $\mathrm{n}^{\circ} 2$, Société Mathématique de France (1996), xiv + $251 \mathrm{pp}$.

[12] V. Toulmonde, Module de continuité de la fonction de repartition de $\varphi(n) / n$, Acta Arith. 121 $\mathrm{n}^{\mathrm{o}} 4(2006), 367-402$.

[13] V. Toulmonde, Sur les variations de la fonction de répartition de $\varphi(n) / n, J$. Number Theory $120 n^{\circ} 1$ (2006), 1-12.

[14] V. Toulmonde, Vincent Comportement au voisinage de 1 de la fonction de répartition de $\varphi(n) / n$, Int. J. Number Theory $\mathbf{5} \mathrm{n}^{\mathrm{o}} 8$ (2009), 1347-1384.

Gérald Tenenbaum

Institut Élie Cartan

Université Henri Poincaré-Nancy 1

BP 239

54506 Vandœuvre Cedex

France

internet: gerald.tenenbaum@iecn.u-nancy.fr 